The Parkes telescope in Australia detected the first fast radio burst in 2001. black holes, colliding neutron stars and enormous magnetic eruptions. But even the best model fails to account for all the observations, says Edo Berger, an astronomer at Harvard University in Cambridge, Massachusetts, who describes the situation as "a lot of swirling confusion".

Clarity may come soon, however. Telescopes around the world are being adapted to look for the mysterious bursts. One of them, the Canadian Hydrogen Intensity Mapping Experiment (CHIME) near Penticton in British Columbia, should see as many as a dozen FRBs per day when it comes online by the end of 2017.

"This area is set to explode," says Bailes.

\section{CURIOUSER AND CURIOUSER}

Astronomers might have had more confidence in the Lorimer burst initially had it not been for a discovery in 2010 by Sarah BurkeSpolaor, who was then finishing her astrophysics $\mathrm{PhD}$ at Swinburne. Burke-Spolaor, now an astronomer at the US National Radio Astronomy Observatory in Socorro, New Mexico, was trawling through old Parkes data in search of more bursts when she turned up 16 signals that shook everyone's confidence in the original ${ }^{3}$.

In most ways, these signals looked remarkably similar to the Lorimer event. They, too, showed 'dispersion', meaning that highfrequency waves appeared in the detectors a few hundred milliseconds before the lowfrequency ones. This dispersion effect was the most important piece of evidence convincing Lorimer and Bailes that the original burst came from well beyond our Galaxy. Interstellar electrons in clouds of ionized gas are known to interact more with lowfrequency waves than with high-frequency ones, which delays the low-frequency waves' arrival at Earth ever so slightly, and stretches the signal (see 'Flight delays'). The delay in the Lorimer burst was so extensive that the wave had to have travelled through a lot of matter much more than is in our Galaxy.

Unfortunately for Lorimer and Bailes' peace of mind, Burke-Spolaor's signals also showed a crucial difference from the original: they seemed to pour in from everywhere, not just from where the telescope was pointing. Dubbed perytons, after a mythical winged creature that casts a human shadow, these bursts could have been caused by lightning, or some human-made source. But they were not extraterrestrial.

Lorimer decided to postpone his research into FRBs for a while. "I didn't yet have tenure," he says, "so I had to go back and do more mainstream projects, just to keep my research moving." Bailes and his team kept going, and upgraded the Parkes detector's time and frequency resolution. In 2013, they turned up four new FRB candidates that resembled the Lorimer burst ${ }^{4}$. But some outsiders remained sceptical that the signals were really coming from space - not least because all the FRBs thus far had been seen by one team using one telescope. "I was desperate for someone else to find them somewhere else," says Bailes.

In 2014, his wish was finally granted. A team led by astronomer Laura Spitler at the Max Planck Institute for Radio Astronomy in Bonn, Germany, published their observations of a burst at the Arecibo Observatory in Puerto Rico5. "I was ridiculously overjoyed," says Bailes.

The Arecibo discovery convinced most people that FRBs were the real deal, says Emily Petroff, who is now an astrophysicist at the Netherlands Institute for Radio Astronomy in Dwingeloo. Yet, as long as the Burke-Spolaor signals went unexplained, they cast a shadow of doubt. "At any talks I would give," says Petroff, "someone would say, 'But what about

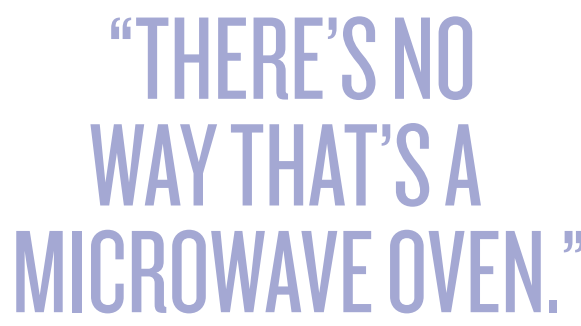

perytons?'" So in 2015, while still a graduate student at Swinburne, she led a hunt to track down the source of perytons once and for all.

First, Petroff and her team used the upgraded Parkes detector to pinpoint when the bursts were happening: at lunchtime. "Immediately I thought, 'This isn't weather," says Petroff. Then came another peryton at a suspiciously familiar radio frequency, which led the team to run experiments in the staff kitchen. Perytons, they discovered, were the result of scientists opening the microwave oven mid-flow. But the Lorimer event was in the clear: records showed that at the time of the burst, the telescope had been pointed in a direction that would have blocked any microwave signal from the kitchen ${ }^{6}$.

"So then I worried, maybe they've just got a different brand of microwave at Arecibo," says Bailes, whose team at Parkes had, by then, racked up 14 separate bursts. He did not relax completely until later in 2015, when a burst was spotted at a third facility - the Green Bank Telescope in West Virginia. That burst had another quality that supported an extraterrestrial origin: its waves were rotated in a spiral pattern - which results from passing through a magnetic field - and were scattered as if they had emerged from a dense medium. "There's no way that's a microwave oven," Bailes told himself.

\section{BURSTS OF INSPIRATION}

But that still leaves the question of what the FRBs actually are. The extreme brevity of the signal, just 5 milliseconds, implied that the source must be a compact object no more than a few hundred kilometres across - a stellar-mass black hole, perhaps, or a neutron star, the compact core left over by a supernova. And the fact that Earth-based telescopes can detect the FRBs at all means that this compact source somehow puts out an immense amount of energy. But that still leaves a long list of candidates, from merging black holes to flares on magnetars: rare neutron stars with fields hundreds of millions of billions of times stronger than the Sun's.

An important clue arrived earlier this year when Spitler's team reported that at least one FRB source repeats: data from Arecibo revealed a flurry of bursts over two months, some spaced just minutes apart ${ }^{7}$. That behaviour has been confirmed by the Green Bank telescope, which detects signals in a different frequency band ${ }^{8}$. Until then, each of the observed FRBs had been a one-off event, which hinted at cataclysmic explosions, or collisions in which the sources were destroyed. But a repeating FRB implies the existence of a source that survives the pulse event, says Petroff. And for that reason, she says, "I would assume it would be something to do with a neutron star" - one of the few known objects that can emit a pulse without self-destructing.

Spitler agrees. As an example, she points to the Crab nebula: the result of a supernova explosion that was observed from Earth in 1054 and left behind a rapidly spinning pulsar surrounded by glowing gas. The Crab pulsar occasionally releases extremely bright and narrow radio flares, Spitler says. And if this nebula were in a distant galaxy and hugely boosted in energy, its emissions would look like FRBs.

If one source repeats, Spitler says, the simplest interpretation is that they all do, but that other telescopes haven't been sensitive enough - or lucky enough - to see the additional signals. Yet others think that perhaps only some are repeating. "I wouldn't be surprised if we end up with two or three populations," says Petroff.

\section{A LONG WAY HOME}

Another crucial question is how far away the FRBs are. The 20 bursts seen so far seem to be scattered randomly around the sky rather than being concentrated in the plane of the Galaxy, which suggests that their sources lie beyond the borders of the Milky Way.

And yet to Avi Loeb, a physicist at Harvard University, such vast distances imply an implausibly large energy output. "If you want the burst to repeat, you won't be able to destroy the source - therefore, it cannot release too much energy," he says. "That puts a limit on how far away you can put it." Perhaps, he says, the FRB sources are neutron stars in our own Galaxy, and the dispersion is mostly the result of still unknown electron clouds that blanket them.

But others suggest that such a dense cloud in the Galaxy should be visible in other wavelengths. At the California Institute of Technology (Caltech) in Pasadena, astrophysicist Shri Kulkarni has scoured data from several telescopes for a galactic source and turned up 
nothing 9 . Kulkarni, who directs Caltech's optical observatories, initially argued for galactic FRBs, and even made a US $\$ 1,000$ bet on it with astronomer Paul Groot of Radboud University Nijmegen in the Netherlands. Now, he finds the evidence for extragalactic FRBs to be overwhelming, and has agreed to settle the bet grudgingly. "I think I will pay him in \$1 bills," he says.

Still, Kulkarni hasn't ruled out the possibility that the FRB sources lie in galaxies that are perhaps a billion light years away, rather than many billions. Such a distance would still require at least some of the signal dispersion to come from electron clouds in the host galaxy, he says. But closer FRBs would not have to be so energetic. "It takes them from being amazingly exotic, to just exotic," he says.

The answer could mean a great deal to observers. If the FRB signals have travelled through local plasma clouds, they could give weather reports from neighbouring galaxies. But if they are truly cosmological - coming from halfway across the visible Universe - they could solve a long-standing cosmic mystery.

For decades, astronomers have known from observations of the early Universe that the cosmos should contain more everyday matter - the kind made up of electrons, protons and neutrons - than exists in the visible stars and galaxies. They suspect that it lies in the cold intergalactic medium, where it is effectively invisible. But now, for the first time, the dispersion of the FRB signals could enable them to measure the medium's density in any given direction. "Then, we have essentially a surgical device to do intergalactic tomography," says Kulkarni.

\section{RAPID-FIRE DETECTION}

First, however, astronomers have to find a lot more FRBs and pin down their locations. "Until then, we are stumbling in the dark," says Berger.

One way to accomplish that is to extract the FRBs from radio-telescope data in real time, so that scientists at other observatories can observe the bursts in multiple wavelengths. Since last year, the Parkes team has been doing this by boosting the observatory's in-house computing power, and scientists at Arecibo hope to follow suit this year. In February, the strategy seemed to be paying off when an independent team followed up within two hours of an FRB's detection at Parkes. The team tentatively pinpointed the burst to a specific galaxy almost 6 billion light years away. Further observations cast doubt on that interpretation. But even so, says Lorimer, the method is sound and may pay off in the future.

Others observers are putting their hopes in new telescopes. In 2014, astrophysicist Victoria

\section{FLIGHT DELAYS}

Astronomers are not sure what causes fast radio bursts (FRBs). But as the waves reach Earth, low-frequency ones lag behind high-frequency ones. The extent of this delay suggests that the signals have travelled through intergalactic space for potentially billions of light years.

\section{SOURCE}

An unknown event

emits a huge burst

of radio waves over

a range of

frequencies

simültaneously.

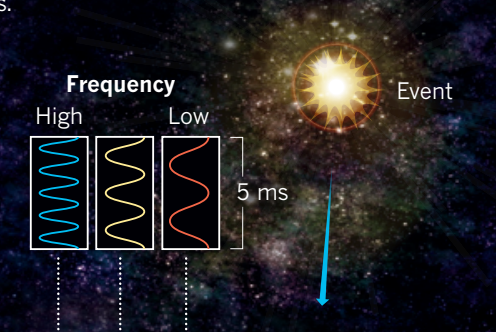

Electron clouds between the

galaxies interact

with the waves,

stretching and

slowing the lowe
slowing and

frequencies more

strongly than the

higher ones.
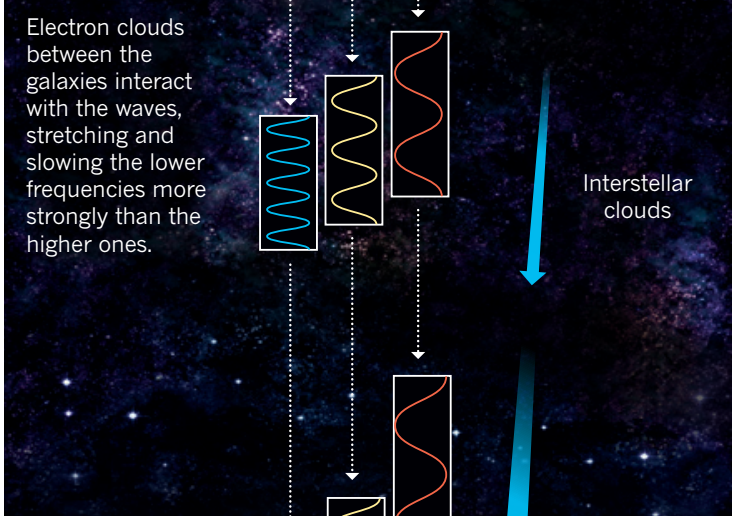

A telescope on Earth measures

the delay and

stretch, enabling

astronomers to

estimate how far

the signals have

the signals have

come.

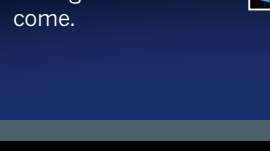

\section{SIENAL}

The signal is lost in

the noise until the

telescope's output

is separated into

frequency bands.

frequency bands
This reveals a

cascade of peaks

that corresponds

to the dispersion of

the burst.

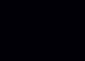

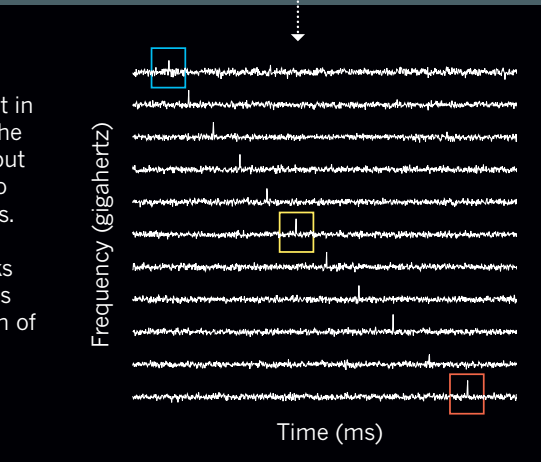

next year, says Kaspi, ultimately finding more than a dozen per day.

In Hoskinstown, Australia, meanwhile, Bailes and his colleagues are refurbishing the 1960s-vintage Molonglo Observatory Synthesis Telescope, turning it into an FRB observatory with a single half-pipe 16 times longer than CHIME's, although one-quarter as wide. The team has already found three as-yet-unpublished FRBs with the facility working at only about $20 \%$ of its final sensitivity, says Bailes.

Another strategy for locating the FRB sources is to work with existing facilities such as the Very Large Array: an 'interferometer' that uses the time difference between signals from 27 radio telescopes spaced across 36 kilometres of grassland near Socorro, New Mexico, to create a single, high-resolution image. Sometime in the next year or so, says Lorimer, the array could detect an FRB and locate its home galaxy. "Ultimately, that could settle a lot of arguments and bets," he says.

Kulkarni, meanwhile, is leading two projects. The first uses ten 5-metrewide dishes in an array that can see and locate only super-bright FRBs, but that makes up for its low sensitivity by peering at a huge swathe of sky. The second takes the principle to the extreme, using 2 antennas spaced at observatories 450 kilometres apart that will see only the very brightest FRBs, but that are able to examine half the sky at once. That would enable it to catch the rare FRBs that presumably exist within our own Galaxy, but whose extreme brightness existing telescopes are not designed to see. "Most facilities would just discount it as interference," says Kulkarni.

If FRBs do turn out to come from cosmological distances, says Loeb, their identification would be a major breakthrough, potentially unravelling a new class of source that could be used to probe the Universe's missing matter. But then, he says, FRBs could also be something that no one has thought of yet: "Nature is much more imaginative than we are." -

Elizabeth Gibney is a reporter for Nature in London.

Kaspi at McGill University in Montreal, Canada, submitted a proposal to adapt CHIME, which was originally designed to map the expansion of the Universe in its early years. "It became clear very quickly that it would be a fantastic FRB instrument," says Kaspi. Although dish telescopes such as Arecibo can be highly sensitive, they observe only a single, tiny patch of sky at a time. CHIME, by contrast, consists of four 100-metre-long half-pipes dotted with antennas that can monitor much bigger stretches of sky in long lines. After undergoing testing and debugging, CHIME should see its first FRBs sometime
1. Lorimer, D. R., Bailes, M., McLaughlin, M. A. Narkevic, D. J. \& Crawford, F. Science 318, 777-780 (2007).

2. Champion, D. J. et al. Mon. Not. R. Astron. Soc. Lett. 460, L30-L34 (2016).

3. Burke-Spolaor, S., Bailes, M., Ekers, R., Macquart, J.-P. \& Crawford, F. III Astrophys. J. 727, 18 (2011)

4. Thornton, D. et al. Science 341, 53-56 (2013).

5. Spitler, L. G. et al. Astrophys. J. 790, 101 (2014)

6. Petroff, E. et al. Mon. Not. R. Astron. Soc. 451, 3933-3940 (2015).

7. Spitler, L. G. et al. Nature 531, 202-205 (2016).

8. Scholz, P. et al. Preprint at http://arxiv.org/ abs/1603.08880 (2016).

9. Kulkarni, S. R., Ofek, E. O. \& Neill, J. D. Preprint at http://arxiv.org/abs/1511.09137 (2015). 


\section{CLARIFICATION}

In the News Feature 'Mystery in the heavens'

(Nature 534, 610-612; 2016), the discussion of the initial radio burst meant to say that over the course of just a few milliseconds, the source's output matched that of 500 million

Suns in the same time period. 